\title{
APPLICATION OF A NEW RAPID TRANSIT NETWORK DESIGN MODEL TO BUS RAPID TRANSIT NETWORK DESIGN: CASE STUDY ISFAHAN METROPOLITAN AREA
}

\author{
Shahab Kermanshahi, Yousef Shafahi, Mehdi Bagherian \\ Dept of Civil Engineering, Sharif University of Technology, Tehran, Iran \\ Submitted 1 November 2011; accepted 12 March 2012; \\ first published online 16 October 2013
}

\begin{abstract}
The problem of Rapid Transit Network Design (RTND) is studied in this paper. Due to the noticeable contribution of rapid transit lines in public transportation network of large urban areas, this problem is interesting to the transportation specialists. On the other hand, the success stories of Bus Rapid Transit (BRT) systems in different countries have motivated us to study BRT network planning. BRT systems can be developed with less investment costs and construction time in comparison with rail-based systems. Therefore, planning Bus Rapid Transit lines, either to develop a new rapid transit network or extend a current one can be an interesting research topic. This problem, like other network design problems is difficult to solve for large scale networks. In this study, a mixed-integer mathematical model that addresses the Transit Network Design Problem (TNDP) is presented. The objective function of the model is maximization of trip coverage. To solve the model, an algorithm is proposed and implemented in C\# environment. The main modules of the algorithm are the following: (1) routes generation, (2) search tree, (3) solution evaluation, and (4) inference. In Route Generation module, the candidate transit route set is determined. Afterwards, the Search Tree module provides a strategy which guarantees that all feasible combinations can be considered in the search process. To evaluate the performance of each transit route combination, a transit assignment algorithm is used in the Solution Evaluation part. Finally, the intelligence core of the search process, that is called Inference, helps the algorithm to find parts of the search space which cannot contain the optimal solution. The algorithm is tested on a real size network, i.e., the extension of the Greater Isfahan rapid transit network with BRT routes. The output of the algorithm is the set of BRT routes that maximizes the daily trip coverage index while satisfying the budget constraint. By solving the case study problem, it is shown that our proposed model and algorithm are capable of tackling real size rapid transit network design problems.
\end{abstract}

Keywords: optimization; bus rapid transit; transit network design; model; algorithm.

\section{Introduction}

Optimization problems in public transit attract researchers' attention due to their size, complexity and practical importance. The problems of optimization of public transit systems are categorized into strategic, tactical, operational, and real-time control levels (Desaulniers, Hickman 2007). At the strategic level, transit route network design is considered while frequency setting and timetabling are usually studied at a tactical level (Bagloee, Ceder 2011). Crew and vehicle scheduling problems are solved in operational planning (Ceder 2002; Bartodziej et al. 2009; Smith et al. 2007). At real-time control level, the main concerned is minimizing the ef- fect of minor and major disruptions in transit networks (Desaulniers, Hickman 2007; Mesa et al. 2013).

Transit route network design at the strategic planning level has been studied by many researchers since 1960s. A comprehensive review of the literature was reported by Kepaptsoglou and Karlaftis (2009). Some researchers noticed the sources of transit route network design complexity (Baaj, Mahmassani 1991; Newell 1979). To make the real world problems smaller and more probable to solve with exact methods, decomposing them into rapid transit and feeder bus network design can be helpful. There are at least 2 reasons which motivate researchers to approach rapid and feeder line

Corresponding author: Shahab Kermanshahi

E-mail: kermanshahi@civil.sharif.ac.ir 
network design problems differently. First, Rapid transit lines usually are long, high capacity lines with dedicated paths while feeder lines connect urban areas to one or more rapid transit stations on mixed traffic paths. Second, rapid transit lines normally pass through freeways and arterial streets, but feeder lines are usually in minor arterials and collector-distributer streets. Feeder lines are supposed to provide better access to rapid transit stations. Once the rapid transit network is planned, appropriate feeder services can be designed to serve it. Regarding this decomposition idea, the Rapid Transit Network Design (RTND) was studied by a number of researchers (Laporte et al. 2007; Marín 2007; Kermanshahi et al. 2010) as an independent problem. The Feeder bus network design was also noticed as an independent problem (Mohaymany, Gholami 2010; Kuan et al. 2006; Shrivastava, O'Mahony 2009), as well.

Laporte et al. (2007) studied and presented a mixed-integer mathematical model for RTND. In their study, they used maximization of the trip coverage as the objective function. Marín (2007) presented an extension of the previous works by relaxing the predefinition of the starting and ending points of rapid transit routes. He used CPLEX to evaluate the model performance on two sample networks with 6 and 9 nodes. Escudero and Muñoz (2009) developed a two-stage method to solve RTND. The first stage resulted in a connected graph that maximized the network coverage. The second stage was designed to extract transit routes from the connected graph. Small networks were used in these former studies. Marín and Jaramillo (2009) applied Accelerated Benders Decomposition to solve the RTND problem. They used the abstract network of the City of Seville with 24 nodes as the case study. As in the former study, the transfer penalty was not considered in his model. Kermanshahi et al. (2010) studied RTND and presented a new model and solution algorithm that considered the transfer penalty. Additionally, a noticeable decrease in computation time in comparison with Escudero and Muñoz (2009) was reported.

In all aforementioned RTND studies, maximization of the covered trips is the objective function. It forces the model to select a combination of rapid lines which offer an acceptable service to as many trips as possible. A transit service is acceptable if its travel time is smaller than a predefined value. In this study, maximizing trip coverage is selected as the objective function. Furthermore, a new concept of target travel time is defined in this study which addresses the maximum acceptable travel time for each transit trip regarding its origin and destination.

In a recent study by Hensher and Golob (2008), it was concluded that connectivity and frequency have a considerable effect on attracting public transit demand; from this viewpoint, the BRT system is in the right place. BRT is one of the most attracting transit systems and can be categorized as a rapid or semi-rapid transit system (Vuchic 2007). BRT is defined as '... a high-quality bus based transit system that delivers fast, comfortable, and cost-effective urban mobility through the provision of segregated right-of-way infrastructure, rapid and frequent operations, and excellence in marketing and customer service' (Wright, Hook 2007). It is a leading transit system for improvement and development of transit networks in numerous cities all over the world (Deng, Nelson 2011; Cain et al. 2007; Polzin, Baltes 2002). Its lower construction cost in comparison with rail-based systems makes it more attractive for decision makers. RTND can help extending the rapid transit network of cities using BRT lines. So, we were motivated to develop a new model and algorithm to solve BRT network design problem in a real-size network.

A new mathematical model for Transit Network Design Problem (TNDP) is presented in the problem formulation section. Following that the solution algorithm modules are illustrated. The modules are described in four subsections: route generation, search tree, solution evaluation, inference. Next, the problem of extending the of greater Isfahan rapid transit network using BRT routes is solved and computational results of different experiments are reported in the case study section. Finally, the paper's findings are reflected in the concluding section.

\section{Problem Formulation}

The TNDP can be stated as a discrete combinatorial optimization problem: The selection of some transit routes from a given candidate set of routes that maximize the defined coverage index while satisfying the budget constraints. It is assumed that a trip is covered with the transit system when the travel time provided by the transit system is not greater than a predefined value. We called this predefined value the Target Travel Time. The main inputs of the model are the set of candidate routes, demand matrix, and Target Travel Time matrix. A set of binary variables, each of which corresponds to a candidate route, makes up the decision variable array. Another important input parameter of the model is the available budget. The problem can be formulated as follows:

$$
\begin{aligned}
& \max z=\sum_{w} c^{w} \cdot g^{w} ; \\
& \sum_{l} y^{l} \cdot c^{l} \leq B, \forall l \in L ; \\
& x_{i j}^{l} \leq \delta_{i j}^{l} \cdot y^{l} \cdot M, \forall i j \in E, \forall l \in L ; \\
& T^{w}-c^{w} \cdot t^{*} w+M \cdot\left(1-c^{w}\right) \geq 0, \forall w \in W ; \\
& t^{*} \text { is obtained from transit assignment } \forall w \in W,
\end{aligned}
$$

where: $c^{w}$ - binary variable of coverage, which is 1 if $w$ is covered and 0 , otherwise; $g^{w}$ - number of trips between $w$ OD pair; $y^{l}$ - binary variable of selecting route $l$ which is 1 if route $l$ is selected and 0 , otherwise; $c^{l}$ - construction cost of route $l ; B$ - budget level; $x_{i j}^{l}$ - if link $i j$ belongs to route $l$ and route $l$ is selected in the solution; $\delta_{i j}^{l}$ - is 1 if link $i j$ belongs to route $l$ and 0 , otherwise; $T^{w}$ - target travel time between $w$ OD pair; $t^{*} w$ - transit travel time; $\mathrm{M}$ - a big enough number. 
The definition of trip coverage used in this model is a generalization of the definition of Laporte et al. (2007). Our definition considers the provision of an acceptable transit service that offers an acceptable generalized travel time (including access time, egress time, waiting time, transfer penalty and in-vehicle time) to OD passengers. It seems that the target travel time of a given OD pair may depend on many parameters such as the travel time by way of private car between them, the socio-economic characteristics of the passenger and the trip purpose, characteristics of origin and destination as well as the current provided transit travel time. In this study, it is assumed that the target travel time matrix between OD pairs is given, but it may be valuable to study passenger preferences to generate a target travel matrix, probably for different passenger market segments.

Equation (1) represents the objective function that is the summation of covered trips over all OD pairs. The constraint in equation (2) stands to guarantee that the solution is budget feasible. The construction cost of each single route is calculated at route generation procedure (section 2.1). The constraints in equation (3) guarantee that if a route is not selected, it is not used by transit passengers. The constraints in equation (4) reflect the coverage definition for an OD pair $w$; if the provided service by transit system $t^{*} w$ is greater than $c^{w}$ (the target travel time of $w$ ), then the corresponding coverage binary variable, is forced to be zero. Finally, $t^{*} w$ is obtained from the transit assignment procedure and stated in equation (5). The procedure of transit assignment is described in section 2.3. Some of the major constraints of BRT route layout and passengers movement in the transit network are not presented in this formulation. Indeed, route layout related constraints are considered implicitly in the route generation module. Besides, flow conservation constraints are embedded in transit assignment (Eq. 5).

\section{Solution Algorithm}

To solve the model presented in the previous section, an algorithm is developed based on implicit enumeration and is implemented in $\mathrm{C} \#$ environment. The main modules of the algorithm are the following:

- Route Generation;

- Search Tree;

- Solution Evaluation;

- Inference.

In Fig. 1, a flowchart of the algorithm is shown.

\subsection{Route Generation}

The purpose of the proposed TNDP is to find the optimal routes from a set of candidate routes. To identify candidate routes, a Route Generation module is designed. This module generates routes between predefined terminals by selecting a sequence of links on the given base network. In this procedure sub-tours in the generated routes are avoided and routes with sub-tours are not included in the candidate set. The base network includes links that are appropriate for transit vehicles to pass through. Two constraints in Route Generation

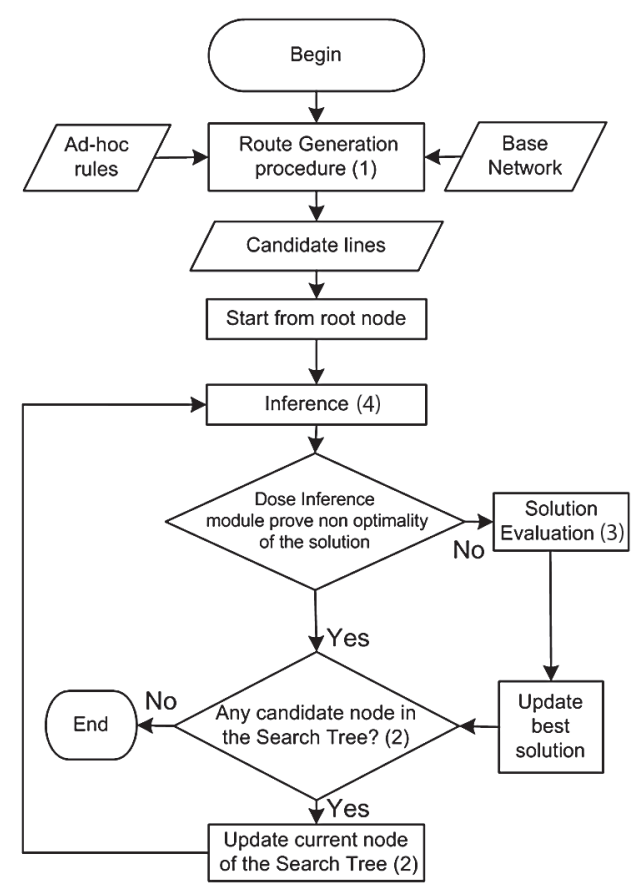

Fig. 1. Solution algorithm flowchart

module are considered. First, the length of routes should be between a given minimum and maximum amount. Second, the acceptable routes should be direct, i.e., they cannot be longer than $(1+\alpha)$ times the shortest distance between their terminals on the base network. These constraints are embedded in the route generation module and do not appear in the model formulation explicitly.

\subsection{Search Tree}

The Search Tree is developed to search through different combinations in a systematic manner to benefit from the information that is gathered through the search process. A schematic search tree is shown in Fig. 2. In the search tree, each node corresponds to a route combination (a subset of a candidate route set). In this figure, the bold path, which starts at the root and passes nodes 1, 4 and 6 , shows a combination of the following three routes: 1 , 4 and 6 . Therefore, at each node, the nodes on the back track connecting it to the root show the corresponding combination. When a branch cannot grow, a reverse movement on the tree is necessary to jump to the next unseen node. The search process terminates when there are no unseen nodes in the search tree.

\subsection{Solution Evaluation (SE)}

The transit travel time between each $w$ OD pair is obtained from transit assignment (Equation (5) in the problem formulation). In this study, it is assumed that passengers choose the shortest path in the transit network to reach their destination. The following two major assumptions are made:

- the transit travel time of each route is independent from the transit volume using it;

- the capacity constraint of transit routes is not considered. 
a)

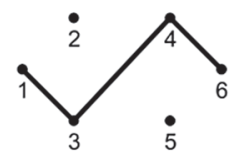

Candidate line 1

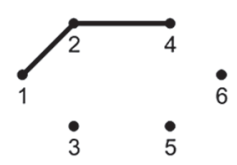

Candidate line 4

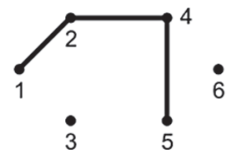

Candidate line 2

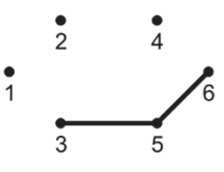

Candidate line 5

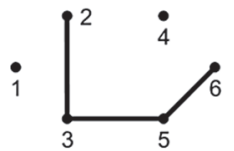

Candidate line 3

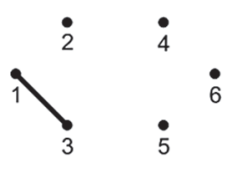

Candidate line 6

b)

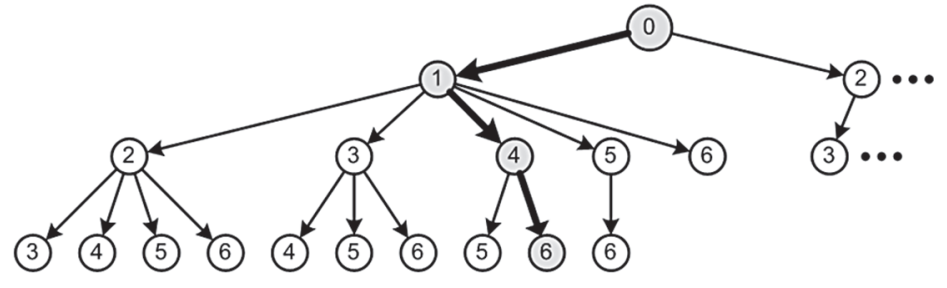

The selected path corresponds to combination of line numbers 1,4 and 6

Fig. 2. Schematic search tree

To solve the shortest path problem on the transit network, the Floyd-Warshall algorithm (Ahuja et al. 1993) is applied. Because it is important in the transit network to consider the waiting time at boarding and the transfer penalty (when a transfer between transit routes occurs), a network transformation procedure is used:

Step 1: each $s_{k}$ rapid station on the initial network is replicated to nodes $s_{k}^{a}$ and $s_{k}^{b}$, which respectively denote alighting and boarding at station $s_{k}$ (see Fig. $3 \mathrm{~b}$, nodes $2 \mathrm{a}$ and $2 \mathrm{~b}$ ).

Step 2: each $s_{k}$ rapid station on the initial network is replicated $\mathrm{n}$ times (to the maximum number of routes in each of the possible combinations). These nodes are denoted as the following: $s_{k}^{1}, s_{k}^{2}, \ldots, s_{k}^{n}$ (see Fig. $3 \mathrm{c}$, nodes 2-1 and 2-2).

Step 3: The candidate routes in the given combination are sorted and each route is set up on its corresponding replicated node, i.e. the $i$-th route may be positioned on (see Fig. 3d; route 1 is positioned on node $2-1$ and route 2 on $2-2$ ).

Step 4: $s_{k}^{a}$ and $s_{k}^{b}$ are connected to $s_{k}^{1}, s_{k}^{2}, \ldots, s_{k}^{n}$ with proper one-way links. For boarding links, the cost is equal to the related route waiting time and for alighting links, the cost is zero. Additionally, $s_{k}^{a}$ and $s_{k}^{b}$ are connected to each other with a one-way link, which denotes a transfer penalty $s_{k}^{i}$ (see Fig. 3e; WT1 and WT2 are the link costs of boarding links from $2 \mathrm{~b}$ to $2-1$ and 2-2; link from 2a to $2 \mathrm{~b}$ has TP cost).

Step 5: for each $s_{k}$ station, outgoing trips are assigned to the corresponding boarding node $s_{k}^{b}$ and incoming trips to the alighting node $s_{k}^{a}$ (see Fig. 3f; outgoing trips from node 2 are assigned to node $2 \mathrm{~b}$; incoming trips are assigned to node $2 \mathrm{a}$ ).

\subsection{Inference}

Design of faster algorithms is valuable because they help solving larger problems in faster and save time on analysis. To do that, recognition of parts of the search space that cannot reach the optimal solution is a possible strategy. In the proposed algorithm, all the tools which help the search process know more about the search space are included in the Inference module. Albeit the algorithm can work without Inference Module, its implementation can accelerate the algorithm. The efficacy of this module is evaluated in the computational results section of the case study problem. The followings are the Inference Module components:

1. Budget infeasibility: Each branch of the search tree may grow until it is cut on the basis of branch cutting criteria. If a combination's cost exceeds the budget level, it is infeasible and none of the predecessors of the corresponding node in the search tree can be feasible. Therefore, the branch should be cut at this node. After branch cutting, a back-track movement is needed to continue the search process.

2. Extendable routes: A route is considered extendable if a link can be added to the route and the extended route still belongs to the candidate route set. Obviously, the coverage index of the extended route cannot be lower than the initial route. If an extendable route $l_{m}$ is in a given $c_{n}$ combination with enough budget for extending it, then there is another $c_{n}^{\prime}$ combination in which $l_{m}$ is replaced by its extended $l_{m}^{\prime}$ route, and this combination provides coverage that is greater than or at least equal to that provided by $c_{n}$. So, it is not necessary to evaluate $c_{n}$ in SE module. 
a)

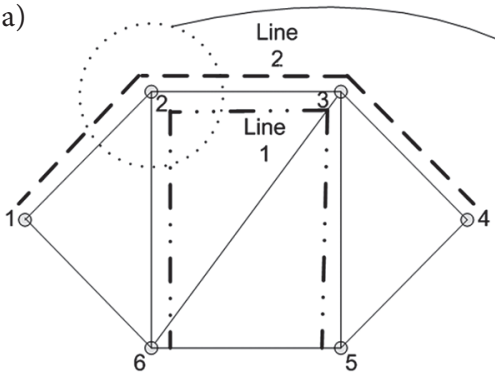

d)

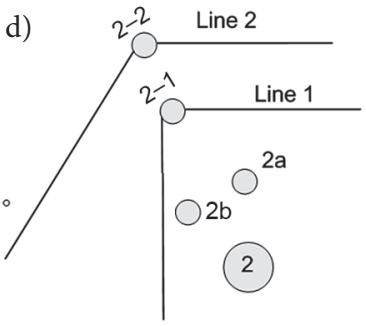

b)<smiles>C1CC1</smiles>

$2 \mathrm{~b} O$

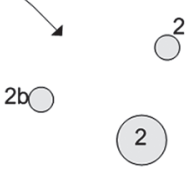

(2) c)

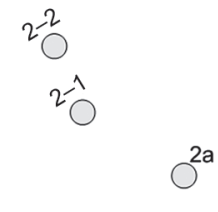

2

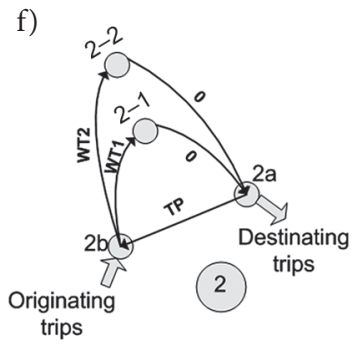

Fig. 3. Network transformation steps on a small network

3. Free transfer network: If the transfer penalty and the waiting time are supposed to be zero, then SE may overestimate the coverage (Kermanshahi et al. 2010). If the transfer penalty is ignored, then a simpler presentation of the network is possible (Fig. 4). Replicated nodes are reduced in this network presentation and a considerably lower computation time can be expected. In this presentation, some BRT route combinations may lead to the same free transfer network (presented networks in Figs $4 \mathrm{a}$ and $4 \mathrm{~b}$ have the same free transfer network as shown in Fig. 4c). Therefore, one single computation for a transfer free network may be used for several route combinations. The objective value can be saved in a lookup table and called whenever necessary.

4. Recognition of transfer-necessary trips: The calculated upper bound in the transfer-free network can be tightened by identifying trips that cannot be served without a transfer between rapid transit routes. If the origin and destination of $w$ OD pair are not located on the same route, then each trip requires at least one transfer to be completed. Accordingly, a distance matrix that is derived from the previous part, (i.e. free transfer network) can be modified and a tighter upper bound can be achieved.

\section{Case Study}

The proposed model and algorithm were applied to the Isfahan metropolitan road network. Currently, about 1000 buses are operating on 90 routes in the metropolitan area transit system. In the transportation master plan, a rapid transit system consisting of three LRT routes is approved (Fig. 5). LRT1 is under construction and the detailed design of LRT2 is in process. Furthermore, BRT1 route, which is approximately $17 \mathrm{~km}$ in length and connects a southwest terminal to a northeast one, is under construction. To extend the current rapid transit network of Greater Isfahan, additional BRT lines were considered. Therefore, only BRT lines are in the routes candidate set for the RTND problem. In other words, our goal is to extend rapid transit network of Greater Isfahan using BRT lines. Rail based lines were planned earlier (55.3 km length in 3 LRT lines) and now our problem is to extend it by a lower cost system: BRT.

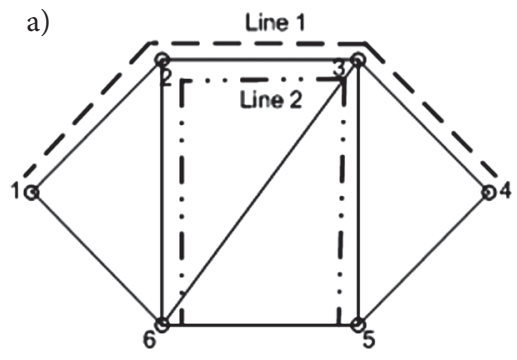

Line 1: 1-2-3-4, Line 2: 6-2-3-5 b)

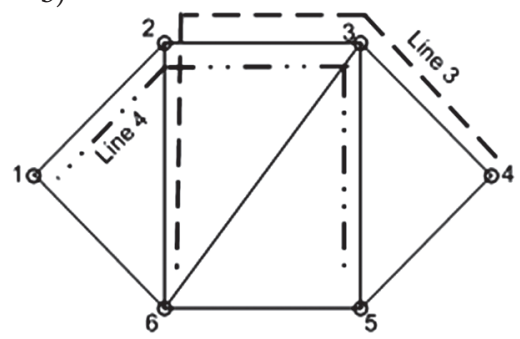

Line 3: 6-2-3-4, Line 4: 1-2-3-5 c)

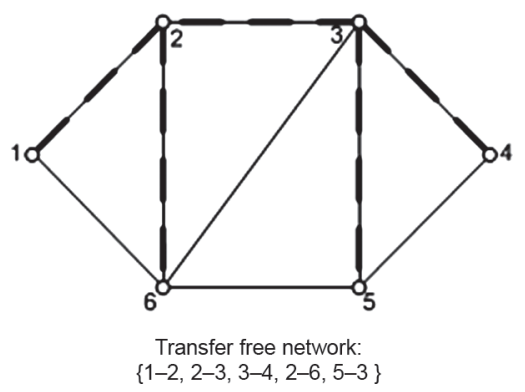

Fig. 4. Transfer-free network 


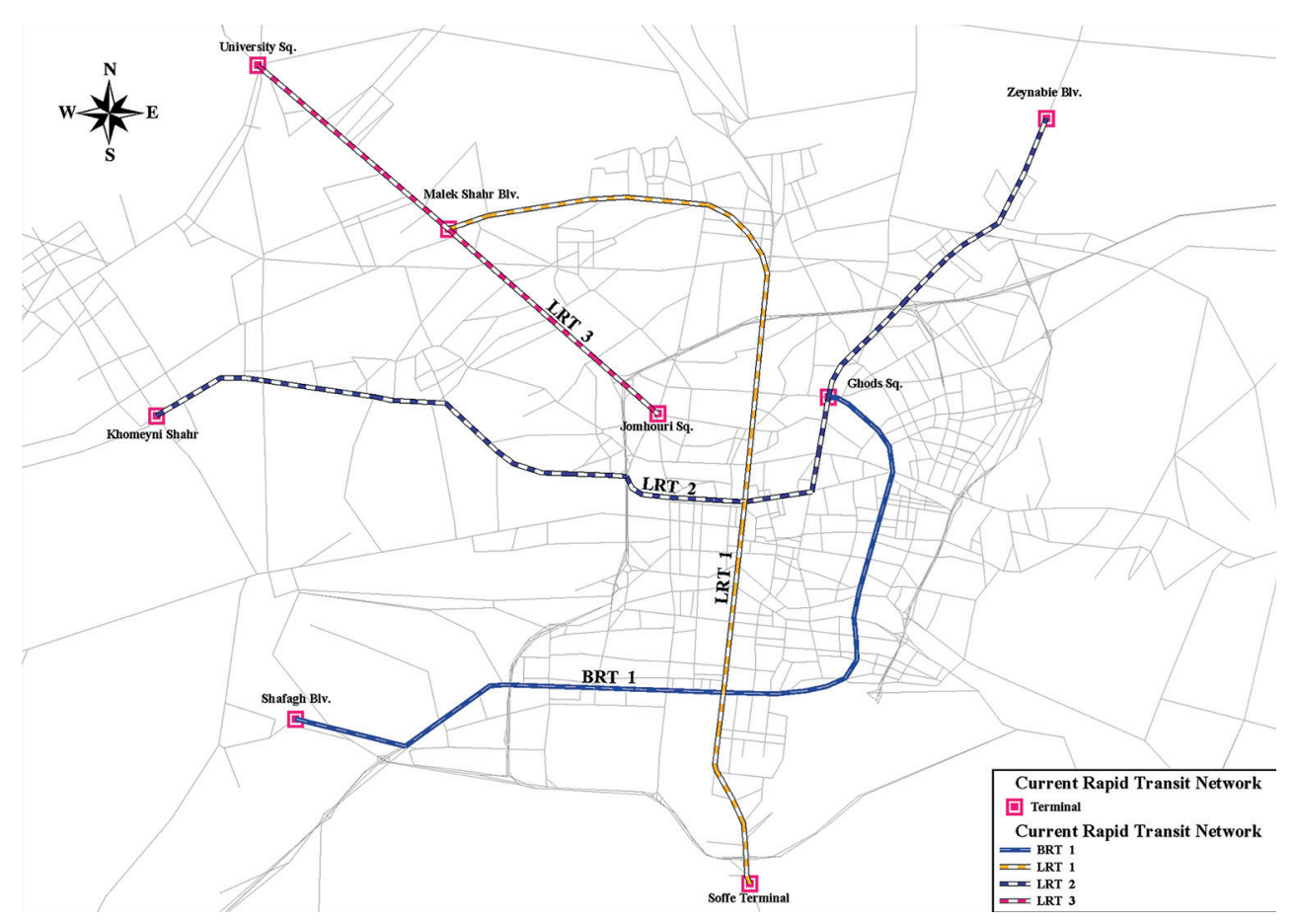

Fig. 5. Greater Isfahan rapid transit network

In the following sub-sections the Input of the model is described in three different parts:

- BRT System specifications;

- Network information;

- Demand Information.

The Input data files are also available at http://www. mehdibagherian.com

\subsection{BRT System Specifications}

The construction cost of BRT routes depends on the selection of the system's main features, including the path type, vehicle type, fare collection method, station facilities, and ITS. It can be calculated for each route independently according to its specifications. In this study, the average construction cost per $\mathrm{km}$ was supposed to be USD 2 million based on certain Asian and Latin American systems (Wright, Hook 2007) that are more applicable to developing countries. This value was used to calculate routes construction costs at route generation procedure (section 2.1). Furthermore, minimum and maximum input values for BRT routes' length were assumed to be 8 and $15 \mathrm{~km}$, respectively. The directness ratio was supposed to be 1 ; thus the shortest path between each terminal pair corresponded to a BRT route. Applying these constraints, 56 BRT routes were generated by Route Generation Module and composed the candidate routes set.

To prepare the network for executing the algorithm, the following assumptions are made. The auto assignment target travel time matrix, resulted from multiplying the predicted private car travel time matrix by a scalar value of 2 . To consider the transit travel time, BRT and LRT routes speeds are assumed to be $30 \mathrm{~km} / \mathrm{h}$ and $40 \mathrm{~km} / \mathrm{h}$, respectively.

\subsection{Network Information}

A base network that is a subset of the transportation network is shown in Fig. 6. It contains arterials that are physically appropriate for BRT vehicles to pass. In this network, a set of 113 rapid transit stations, including 14 given terminals, are selected. BRT routes can start and end only in terminal stations.

\subsection{Demand Information}

From the predicted 6240000 daily trips in the metropolitan area in horizon year, 2148000 are started and ended in our study area being presented in a $180 \times 180$ OD matrix (initial segmentation). A transformation procedure was needed to convert the $180 \times 180$ daily OD matrix to a new matrix which its elements were potential trips between rapid stations (secondary segmentation). In this procedure, two assumptions were made:

1. each trip is assigned to its nearest station;

2. traffic analysis zones are homogenous.

An illustrative example is presented in Fig. 7. In this figure, $P$ zones $\left(P_{i}, P_{j}, P_{k}, P_{l}\right)$ and $Q$ zones $\left(Q_{m}, Q_{n}\right)$ are from the initial and the secondary segmentations of the area, respectively. The OD-matrix components of secondary segmentation can be written as:

$$
\begin{aligned}
& t^{m n}=\sum_{i} \sum_{j} t_{i j}^{m n}, \\
& i, j \in \text { initial segmentation, } \\
& m, n \in \text { secondary segmentation. }
\end{aligned}
$$




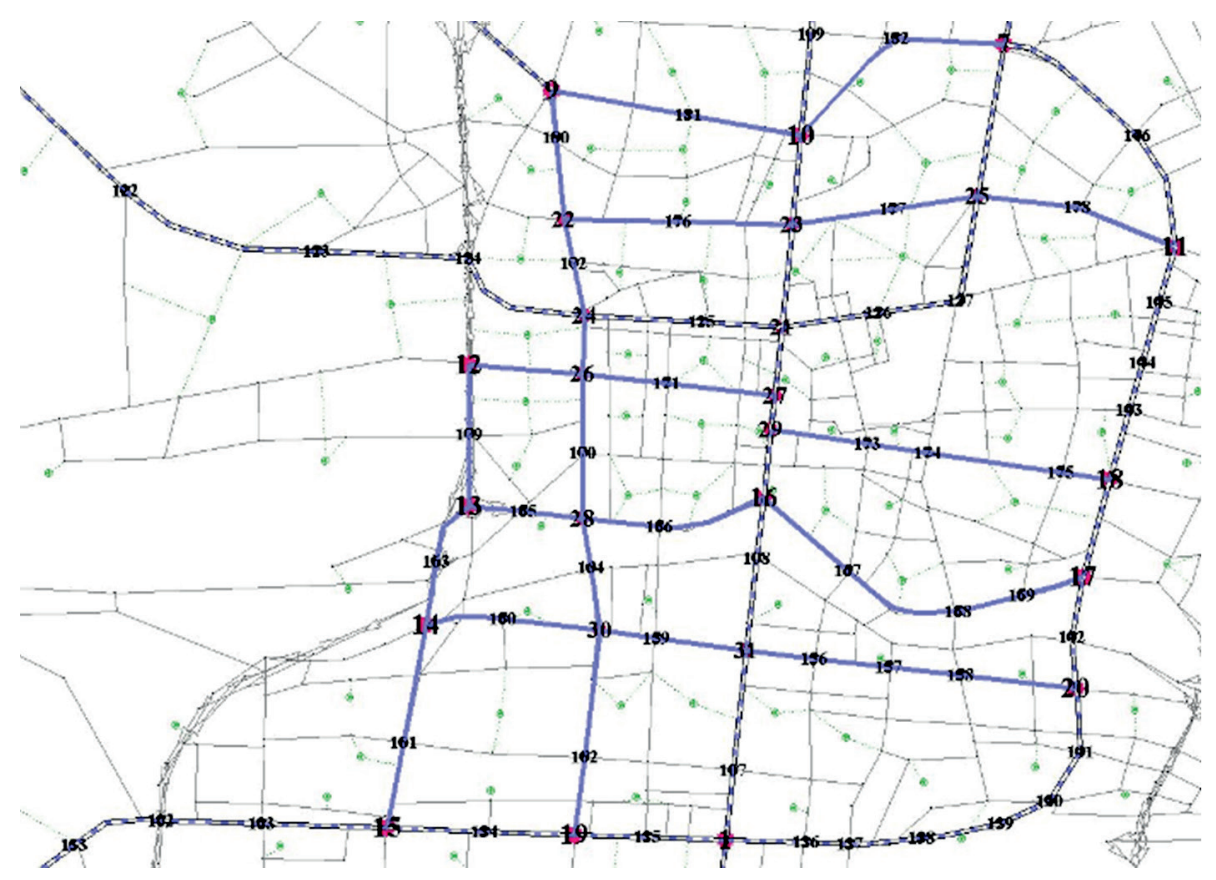

Fig. 6. Base rapid transit network

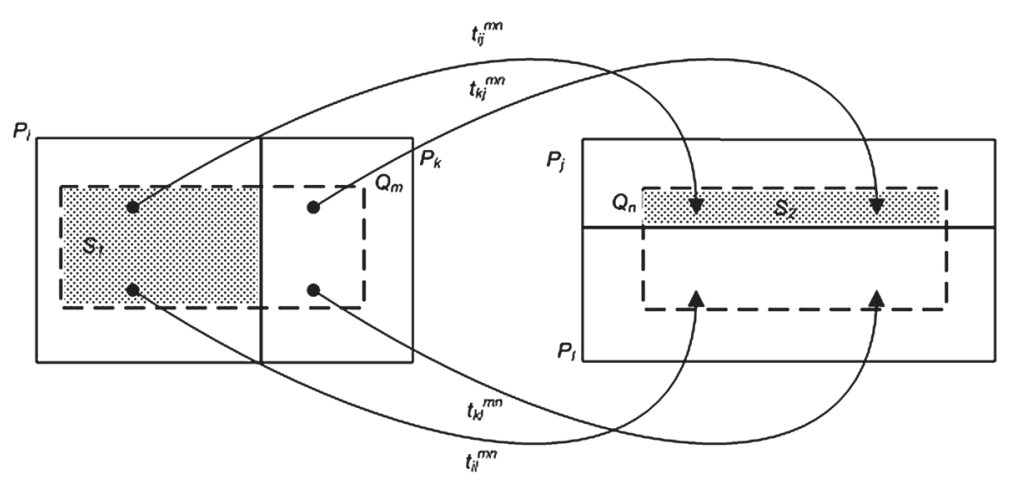

Fig. 7. Illustration of OD-matrix transformation

In the illustrated example,

$t^{m n}=t_{i j}^{m n}+t_{i l}^{m n}+t_{k j}^{m n}+t_{k l}^{m n}$. Also from the homogeneity assumption, $t_{i j}^{m n}=t_{i j} \cdot \frac{S_{1}}{S\left(P_{i}\right)} \cdot \frac{S_{2}}{S\left(P_{i}\right)}$, where $S\left(P_{i}\right)$ is the area of polygon $P_{i}$. This equation is the base of ODmatrix transformation procedure.

\section{Computational Results and Sensitivity Analysis}

The model was solved on this network and a brief description of the model output under different budget scenarios is presented in Table 1. As shown in the table, the objective function increases together with the budget level. The computational time is affected by budget level as well; the higher the budget level the larger the search area and therefore, the longer the computation time. The results show that the CPU time increased as much as 3700 times when the budget was increased from USD 30 million to USD 120 million.
In Table 1, it can be seen that increasing the budget level from USD 30 million to USD 120 million, increases the coverage from $67.2 \%$ to $73.8 \%$. The increasing trend is as expected; the more money we invest the more trips we can cover. Noting that about $63 \%$ of trips can be covered by the existing plan of rapid transit systems, it can be seen that every additional coverage percent, costs about USD 11.1 million in infrastructure $(10.8 \%$ increase for USD 120 million investment). In Fig. 8, the optimal network for a budget of USD 120 million is shown.

To evaluate the efficiency of the Inference module, its effect on CPU time is analysed by two experiment sets. In the first, the inference module is included in the algorithm, in the second it is removed (Table 2). It is noticeable that the algorithm speed may increase up to 10 times when this module is used. Additionally, the Inference module efficiency seems to be more remarkable in larger problems (Table 2). 
Table 1. Model outputs under different budget scenarios

\begin{tabular}{|c|c|c|c|c|}
\hline Budget [million USD] & Objective function & Optimal combination & CPU time $[\mathrm{s}]$ & Coverage ratio [\%] \\
\hline 30 & 1444856 & Route 5: 6-9-22-24-26-12-13-14-15 & 0.2 & 67.2 \\
\hline 60 & 1499122 & $\begin{array}{l}\text { Route 5: 6-9-22-24-26-12-13-14-15 } \\
\text { Route 17: 2-15-14-30-28-16 }\end{array}$ & 6 & 69.7 \\
\hline 90 & 1558164 & $\begin{array}{l}\text { Route 17: } 2-15-14-30-28-16 \\
\text { Route 23: 5-7-10-9 } \\
\text { Route 25: 6-9-22-24-26-12-13-14 } \\
\text { Route 38: 5-7-11 }\end{array}$ & 130 & 72.5 \\
\hline 120 & 1586499 & $\begin{array}{l}\text { Route 5: 6-9-22-24-26-12-13-14-15 } \\
\text { Route 9: 5-7-11-18-17 } \\
\text { Route 17: } 2-15-14-30-28-16 \\
\text { Route 23: } 5-7-10-9 \\
\text { Route 27: } 3-1-20-17-18-11\end{array}$ & 1112 & 73.8 \\
\hline
\end{tabular}

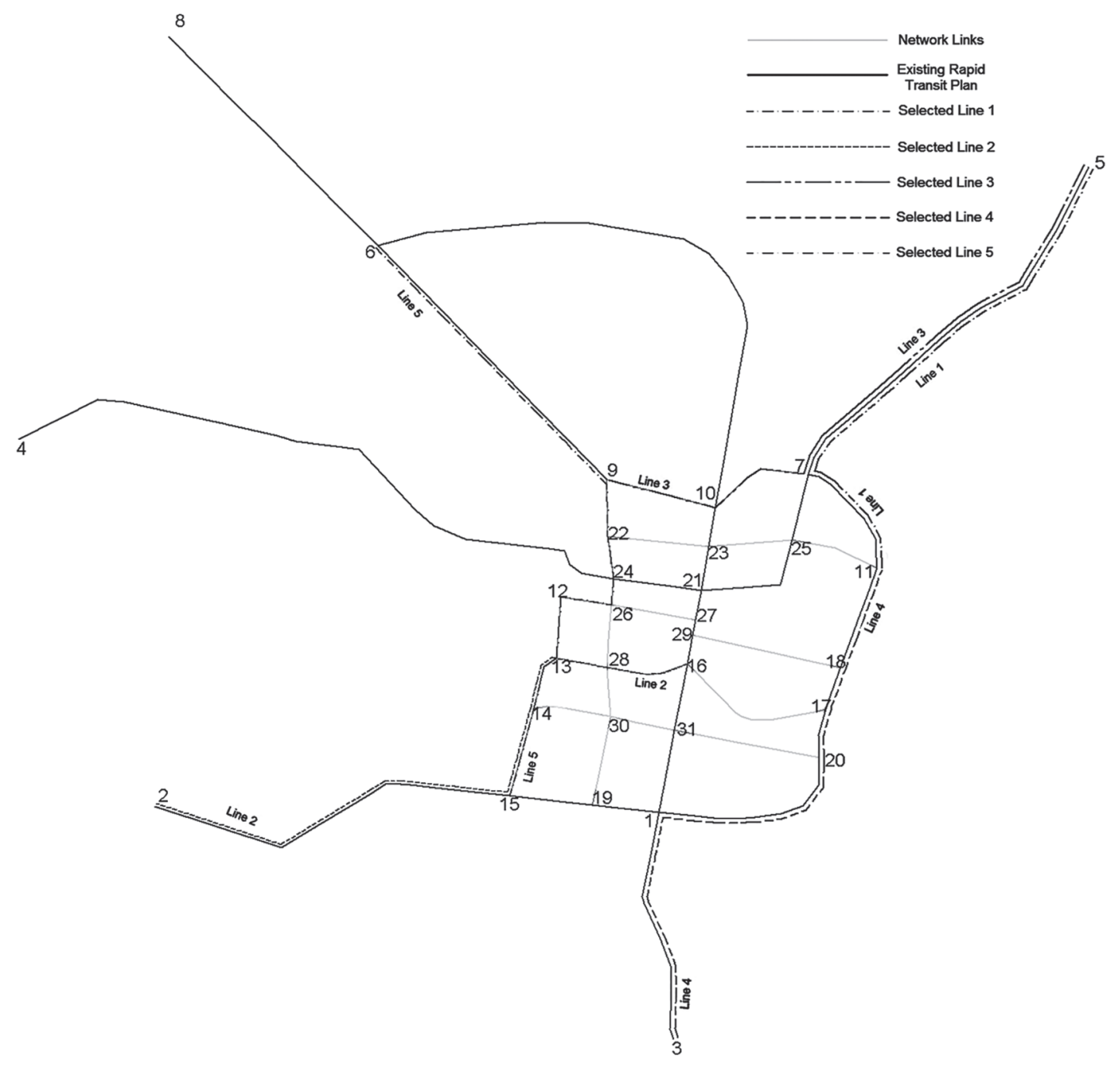

Fig. 8. Optimal network 
Table 2. The effect of inference module on the algorithm's performance

\begin{tabular}{ccccc}
\hline \multirow{2}{*}{$\begin{array}{c}\text { Budget } \\
\text { [million USD] }\end{array}$} & With inference & Without inference & Time saving [\%] & Speed up ratio \\
\cline { 2 - 5 } & 0.2 & 0.2 & 4 & 1.0 \\
\hline 30 & 0.8 & 0.8 & 6 & 1.1 \\
\hline 40 & 3 & 5.8 & 49 & 5.9 \\
\hline 60 & 6 & 12.5 & 52 & 3.1 \\
\hline 70 & 24 & 86 & 71 & 4.0 \\
\hline 80 & 38 & 149 & 84 & 6.2 \\
\hline 90 & 130 & 807 & 86 & 7.1 \\
\hline 100 & 209 & 1492 & 90 & 9.7 \\
\hline 110 & 566 & 5475 & 91 & 10.8 \\
\hline
\end{tabular}

\section{Conclusions}

Due to the noticeable contribution of rapid transit lines in public transportation network of large urban areas, this problem is interesting for the transportation specialists. Over the past years, development of Bus Rapid Transit (BRT) systems has attracted the attention of decision makers in many countries. Developing A model and algorithm for planning a rapid transit network by BRT lines can be interesting. As a result, solving Bus Rapid Transit network design on a real size network was established as the main goal of our study.

In this paper, a new mathematical model for a rapid transit network design problem was presented and applied to a real-size case study. The model is a Mixed Integer Program which aims at finding the best combination of transit routes which maximizes trip coverage. To solve the model, a solution algorithm was developed and coded in C\# environment, consisting of 4 major parts: (1) route generation, (2) search tree, (3) solution evaluation and (4) inference. The algorithm was capable of solving the problem of extending BRT routes on Greater Isfahan network. In this study, solving a rapid transit network problem on a large real network in less than 1 hour was a remarkable achievement. Additionally, applying a set of simple computations to recognize nonoptimal solutions made the algorithm up to 10 times faster. This is interesting from practical viewpoints because large problems like Greater Isfahan BRT network design can be solved in an acceptable amount of time, using the proposed algorithm.

For Greater Isfahan case study, computational results show that by developing new BRT routes, the trip coverage index increases about $10 \%$ with a budget of about USD 120 million. The change in the objective function is interesting because it is obtained from a budget that is only enough to construct a shorter than $6 \mathrm{~km}$ LRT line. It is interesting that a remarkable improvement in transit network performance can be obtained from developing BRT lines with a relatively small investment in construction of routes. Finally, our results verifies previous studies (Hensher, Golob 2008; Wright,
Hook 2007; Deng, Nelson 2011) which recommend BRT as an effective solution to encourage citizens to use rapid transit instead of private cars.

\section{References}

Ahuja, R. K.; Magnanti, T. L.; Orlin, J. B. 1993. Network Flows: Theory, Algorithms, and Applications. Prentice Hall. 864 p.

Bagloee, A. S.; Ceder, A. A. 2011. Transit-network design methodology for actual-size road networks, Transportation Research Part B: Methodological 45(10): 1787-1804. http://dx.doi.org/10.1016/j.trb.2011.07.005

Baaj, M. H.; Mahmassani, H. S. 1991. An AI-based approach for transit route system planning and design, Journal of Advanced Transportation 25(2): 187-209. http://dx.doi.org/10.1002/atr.5670250205

Bartodziej, P.; Derigs, U.; Malcherek, D.; Vogel, U. 2009. Models and algorithms for solving combined vehicle and crew scheduling problems with rest constraints: an application to road feeder service planning in air cargo transportation, OR Spectrum 31(2): 405-429. http://dx.doi.org/10.1007/s00291-007-0110-7

Cain, A.; Darido, G.; Baltes, M. R.; Rodriguez, P.; Barrios, J. C. 2007. Applicability of TransMilenio Bus Rapid Transit System of Bogotá, Colombia, to the United States, Transportation Research Record 2034: 45-54. http://dx.doi.org/10.3141/2034-06

Ceder, A. 2002. Urban transit scheduling: framework, review and examples, Journal of Urban Planning and Development 128(4): 225-244.

http://dx.doi.org/10.1061/(ASCE)0733-9488(2002)128:4(225)

Deng, T.; Nelson, J. D. 2011. Recent developments in bus rapid transit: a review of the literature, Transport Reviews 31(1): 69-96. http://dx.doi.org/10.1080/01441647.2010.492455

Desaulniers, G.; Hickman, M. D. 2007. Public transit, Handbooks in Operations Research and Management Science 14: 69-127. http://dx.doi.org/10.1016/S0927-0507(06)14002-5

Escudero, L. F.; Muñoz, S. 2009. An approach for solving a modification of the extended rapid transit network design problem, Top 17(2): 320-334. http://dx.doi.org/10.1007/s11750-008-0060-5

Hensher, D. A.; Golob, T. F. 2008. Bus rapid transit systems: a comparative assessment, Transportation 35(4): 501-518. http://dx.doi.org/10.1007/s11116-008-9163-y 
Kepaptsoglou, K.; Karlaftis, M. 2009. Transit route network design problem: review, Journal of Transportation Engineering 135(8): 491-505.

http://dx.doi.org/10.1061/(ASCE)0733-947X(2009)135:8(491)

Kermanshahi, S. A.; Shafahi, Y.; Zangui, M.; Mollanejad, M. 2010. Rapid transit network design: a new model and solution algorithm, in TRB 89th Annual Meeting Compendium of Papers DVD, 10-14 January 2010, Washigton, DC. 18 p. (DVD).

Kuan, S.; Ong, H. L.; Ng, K. M. 2006. Solving the feeder bus network design problem by genetic algorithms and ant colony optimization, Advances in Engineering Software 37(6): 351-359. http://dx.doi.org/10.1016/j.advengsoft.2005.10.003

Laporte, G.; Marín, Á.; Mesa, J. A.; Ortega, F. A. 2007. An integrated methodology for the rapid transit network design problem, Lecture Notes in Computer Science 4359: 187-199. http://dx.doi.org/10.1007/978-3-540-74247-0_9

Marín, Á. 2007. An extension to rapid transit network design problem, Top 15(2): 231-241. http://dx.doi.org/10.1007/s11750-007-0017-0

Marín, Á. G.; Jaramillo, P. 2009. Urban rapid transit network design: accelerated Benders decomposition, Annals of Operations Research 169(1): 35-53.

http://dx.doi.org/10.1007/s10479-008-0388-0

Mesa, J. A.; Ortega, F. A.; Pozo, M. A. 2013. A geometric model for an effective rescheduling after reducing service in public transportation systems, Computers \& Operations Research 40(3): 737-746. http://dx.doi.org/10.1016/j.cor.2011.12.003

Mohaymany, A. S.; Gholami, A. 2010. Multimodal feeder network design problem: ant colony optimization approach, Journal of Transportation Engineering 136(4): 323-331. http://dx.doi.org/10.1061/(ASCE)TE.1943-5436.0000110

Newell, G. F. 1979. Some issues relating to the optimal design of bus routes, Transportation Science 13(1): 20-35. http://dx.doi.org/10.1287/trsc.13.1.20

Polzin, S. E.; Baltes, M. R. 2002. Bus rapid transit: a viable alternative?, Journal of Public Transportation 5: 47-69.

Shrivastava, P.; O'Mahony, M. 2009. Use of a hybrid algorithm for modeling coordinated feeder bus route network at suburban railway station, Journal of Transportation Engineering, 135(1): 1-8.

http://dx.doi.org/10.1061/(ASCE)0733-947X(2009)135:1(1)

Smith, J. S.; Karwan, K. R.; Markland, R. E. 2007. A note on the growth of research in service operations management, Production and Operations Management 16(6): 780-790. http://dx.doi.org/10.1111/j.1937-5956.2007.tb00295.x

Vuchic, V. R. 2007. Urban Transit Systems and Technology. Wiley. 823 p.

Wright, L.; Hook, W. 2007. Bus Rapid Transit Planning Guide. New York, Institute for Transportation and Developement Policy. 686 p. Available from Internet: http://www.itdp.org/ microsites/bus-rapid-transit-planning-guide 